\title{
Avaliação nutricional de idosos institucionalizados na Zona Sul de São Paulo
}

\author{
Nutritional assessment of institutionalized elderly in \\ the Southern Region of Sao Paulo \\ Evaluación nutricional de ancianos \\ institucionalizados en la Zona Sur de São Paulo
}

Dalvineia Carvalho Santana

Daiane Soares Macedo

Nyvian Alexandre Kutz

Marcia Maria Hernandes de Abreu de Oliveira Salgueiro

RESUMO: O envelhecimento populacional é um fenômeno mundial e deve ser tratado com prioridade no contexto social no Brasil. Este trabalho avaliou o estado nutricional de idosos institucionalizados utilizando-se a Mini-Avaliação Nutricional e a antropometria. Os resultados mostraram que o risco de desnutrição e desnutrição são condições de evidenciada importância entre os idosos desta instituição. Uma abordagem interdisciplinar da institucionalização é necessária, para melhorar a saúde e qualidade de vida dos idosos.

Palavras-chave: Idoso; Estado nutricional; ILPI. 
ABSTRACT: Population aging is a global phenomenon and should be treated with priority in the social context in Brazil. This study evaluated the nutritional status of institutionalized elderly using the Mini Nutritional Assessment and anthropometry. The results showed that the risk of malnutrition and malnutrition are remarkable importance of conditions among the elderly of this institution. An interdisciplinary approach to institutionalization is necessary to improve the health and quality of life of older people.

Keywords: Elderly; Nutritional status; Homes for the Aged.

RESUMEN: El envejecimiento poblacional es un fenómeno mundial y debe ser tratado con prioridad en el contexto social en Brasil. Este trabajo evaluó el estado nutricional de ancianos institucionalizados utilizando la Mini-Evaluación Nutricional y la antropometría. Los resultados mostraron que el riesgo de desnutrición y desnutrición son condiciones de evidente importancia entre los ancianos de esta institución. Se requiere un enfoque interdisciplinario de la institucionalización para mejorar la salud y la calidad de vida de las personas mayores.

Palabras clave: Ancianos; Estado nutricional; ILPI.

\section{Introdução}

O envelhecimento populacional é hoje um fenômeno universal, característico tanto dos países desenvolvidos como, de modo crescente, dos países em desenvolvimento. Desde as últimas décadas do século passado, o Brasil se depara com um declínio rápido e acentuado da fecundidade, fenômeno sem precedentes na sua história, e que se sobressai mesmo em comparação com outros países. Esse declínio, combinado com a queda da mortalidade, acarretou um processo de envelhecimento populacional (Alves, et al., 2007).

No Brasil, a Lei n. ${ }^{\circ} 8.842$, de 4 de janeiro de 1994, que dispõe sobre a Política Nacional do Idoso, considera idoso o indivíduo que atingir a idade de 60 anos. A Lei n. ${ }^{\circ}$ 10.741, de 10 de outubro de 2003, do Estatuto do Idoso, preconiza várias ações dentro dessa faixa etária. 
De acordo com o Instituto Brasileiro de Geografia e Estatística (IBGE), atualmente existem no Brasil, aproximadamente, 20 milhões de pessoas com idade igual ou superior a 60 anos, o que representa $10 \%$ da população brasileira (Brasil, 2012). Segundo projeções estatísticas da Organização Mundial de Saúde (OMS), no período de 1950 a 2025, o grupo de idosos no país deverá ter aumentado em quinze vezes, enquanto a população total em cinco. Dessa forma, o Brasil ocupará o sexto lugar quanto ao contingente de idosos, alcançando, em 2025, cerca de 32 milhões de pessoas com 60 anos ou mais de idade (WHO, 2011).

Esse envelhecimento populacional ocasiona uma demanda maior por serviços de saúde e outras modalidades de atendimento para idosos, como as Instituições de Longa Permanência para Idosos (ILPI), e indica uma necessidade de atenção aos fatores que possibilitam o bem-estar dos idosos, incluindo o estado nutricional e a qualidade de vida (Ramos, Pizzato, Ettrich, Melnik, \& Goldim, 2012). O processo acelerado de transição demográfica tem sido associado em diversos países ao aumento na demanda por ILPI, e esses fatos têm sido também vivenciados atualmente no Brasil. A correlação multicausal entre a estrutura etária da população e a demanda por ILPI é determinada pelo seu perfil social e de saúde. (Lisboa, \& Chianca, 2012).

A redução da autonomia física e a instabilidade econômica são fatores que podem tornar o idoso cada vez mais dependente. Sendo assim, faz-se necessário que uma pessoa assuma os cuidados desse idoso. Quando essa opção não é possível no núcleo familiar, a institucionalização aparece como opção (Souza, et al., 2013).

O idoso institucionalizado apresenta características peculiares, pois além de apresentar alterações fisiológicas e patológicas que podem ocorrer no processo de envelhecimento, e que acontecem independentemente do ambiente em que ele vive, é marcado por sentimentos de solidão e exclusão familiar, sendo estes alguns dos fatores determinantes para seu ingresso na ILPI. Este cenário requer uma mudança de prioridades com oferta de serviços relacionados à saúde e suporte a esta população (Menezes, \& Bachion, 2012).

A desnutrição é outro problema comum na velhice, sendo considerado o distúrbio mais importante nesta fase da vida. A desnutrição pode contribuir para o aumento da mortalidade, aumento da susceptibilidade às infecções e a redução da qualidade de vida dos idosos (Sousa, Mesquita, Pereira, \& Azeredo, 2014). Em residentes, a desnutrição está associada com comer metade ou menos alimentos servidos em casas de repouso (Papparotto, Bidoli, \& Palese, 2013).

Santana, D. C., Macedo, D. S., Kutz, N. A., \& Salgueiro, M. M. H. de A. de O. (2016). Avaliação nutricional de idosos institucionalizados na Zona Sul de São Paulo. Revista Kairós Gerontologia, 19(Número Especial 22,

“Envelhecimento e Velhice”), 403-416. ISSNe 2176-901X. São Paulo (SP), Brasil: FACHS/NEPE/PEPGG/PUC-SP 
Vários métodos objetivos e subjetivos têm sido utilizados para avaliar o estado nutricional do idoso. Dentre eles, destacam-se os dados antropométricos como Índice de Massa Corporal (IMC), circunferência muscular do braço (CMB), circunferência do braço (CB), dobra cutânea tricipital (DCT), circunferência da panturrilha (CP), MiniAvaliação Nutricional (MAN) que, juntamente com um inquérito alimentar como o recordatório de 24h (R24h), são parâmetros importantes para o diagnóstico precoce do estado nutricional (Paz, Fazzio, \& Santos, 2012).

A alimentação adequada é uma prevenção para a má nutrição do idoso e suas consequentes doenças crônicas, o que influencia na qualidade de vida, que é a percepção que o indivíduo possui de sua posição na vida dentro do contexto da cultura e sistema de valores nos quais ele vive e em relação aos seus objetivos, expectativas, padrões e preocupações (Ramos, Pizzato, Ettrich, Melnik, \& Goldim, 2012).

Assim, o objetivo deste estudo foi avaliar o estado nutricional de idosos numa ILPI da zona Sul de São Paulo.

\section{Metodologia}

Esta pesquisa consiste em um estudo transversal do tipo descritivo-exploratório, tendo sido aprovada pelo Comitê de Ética em Pesquisa do Centro Universitário Adventista de São Paulo, Campus São Paulo, sob o parecer n. ${ }^{\circ} 1.002 .099$ na data de 26/03/2015 e pela direção da Instituição. O estudo foi realizado em uma ILPI, localizada na zona sul do município de São Paulo, no mês de abril de 2015. Trata-se de uma Associação privada, porém conveniada com a Prefeitura de São Paulo. Residiam na ILPI no momento do estudo 55 idosos de ambos os gêneros, sendo que $50 \%$ eram mantidos pela Prefeitura e os demais particulares.

A definição da amostra dependeu da autorização dos idosos por meio da assinatura no Termo de Consentimento Livre e Esclarecido (TCLE). Para aqueles que não possuíam autonomia foram coletadas assinaturas do responsável legal. Os idosos autônomos e não alfabetizados tiveram a autorização coletada através da impressão digital. Foram considerados autônomos os idosos avaliados pela equipe multidisciplinar da instituição. Os que não tinham autonomia nem responsável legal foram excluídos da pesquisa. Participaram da pesquisa 42 idosos, sendo 27 do sexo feminino e 15 do sexo masculino, com idade entre 63 e 98 anos, com estado civil solteiro, casado, separado ou viúvo. 
Para a coleta de dados, foram utilizados dois instrumentos: 1) um questionário elaborado pelos pesquisadores, contendo informações sociodemográficas: idade (anos), gênero (feminino ou masculino), tempo de institucionalização (meses), estado civil (solteiro, casado, separado ou viúvo), e dados de avaliação nutricional objetiva: peso, altura, Índice de Massa Corporal (IMC), circunferência do braço (CB), circunferência da panturrilha (CP) dobra cutânea tricipital (DCT) e circunferência da cintura (CC);

2) Além da antropometria utilizou-se a MAN, método subjetivo para a detecção precoce de risco nutricional especificamente para a população geriátrica (Sousa, Mesquita, Pereira, \& Azeredo, 2014). A MAN consiste em um instrumento de 18 itens, que abrange quatro dimensões: antropometria, avaliação dietética, avaliação global e autoavaliação. Os itens da primeira etapa, que é a da Triagem, se destinam a detecção dos idosos com risco de desnutrição, e com estes indivíduos foi realizado o restante do questionário que é a etapa de avaliação global. Os escores obtidos classificaram os idosos como: estado nutricional adequado, se a soma foi $\geq 24$; risco de desnutrição para valores entre 17 e 23,5; e desnutrição quando a soma for < 17. (Guigoz, Vellas, \& Garry, 1994).

Para aferição do peso, altura, e determinação do IMC, seguiram-se as orientações da OMS (WHO, 1995). A classificação do IMC dos idosos foi realizada de acordo com os pontos de corte propostos, da seguinte forma: $<22 \mathrm{~kg} / \mathrm{m}^{2}$ magreza, 22 $27 \mathrm{~kg} / \mathrm{m}^{2}$ eutrofia; e $>27 \mathrm{~kg} / \mathrm{m}^{2}$ excesso de peso (NSI, 1994). No caso de idosos cadeirantes, ou que apresentavam dificuldades para ficar de pé, foram utilizadas fórmulas de estimativa para peso e estatura (Chumlea, Roche, \& Steinbach, 1985).

A aferição da CB seguiu as recomendações de (Frisancho, 1984). A classificação se deu de acordo com os seguintes parâmetros: desnutrição grave para $\mathrm{CB}<70 \%$; desnutrição moderada de 70 a 80\%; desnutrição leve de 80 a 90\%; eutrofia de 90 a 110\%; sobrepeso de 110 a 120\%; e obesidade >120\% (Blackbum, \& Thornton, 1979).

A CP foi considerada um marcador de desnutrição e uma medida sensível da massa muscular nos idosos. A coleta de dados seguiu as recomendações da OMS (WHO, 1995). Considerou-se um marcador de desnutrição quando os valores da CP foram inferiores a $31 \mathrm{~cm}$ (Chumlea, Roche, \& Steinbaugh, 1985). Para aferir a DCT, seguiram-se as orientações e classificação propostas. Considerou-se desnutrição grave para DCT <70\%; desnutrição moderada de 70 a 80\%; desnutrição leve de 80 a 90\%; eutrofia de 90 a 110\%; sobrepeso de 110 a 120\%; e obesidade $>120 \%$ (Blackburn, \& Thornton, 1979). 
A CC foi medida e classificada de acordo com as recomendações da OMS (WHO, 2000). Considerou-se risco aumentado para doença metabólica quando a CC foi igual ou superior a $94 \mathrm{~cm}$ (homens); e $80 \mathrm{~cm}$ (mulheres); e risco aumentado substancialmente para CC igual ou superior a $102 \mathrm{~cm}$ (homens); e $88 \mathrm{~cm}$ (mulheres).

Todos os dados foram coletados pelo pesquisador e organizados em planilha de Excel. Os resultados foram expressos em média, desvio-padrão, mínima, máxima, frequência simples e relativa.

\section{Resultados}

A amostra foi composta por 42 idosos, dos 55 institucionalizados, com média de idade de 79,5 $\pm 10,1$ anos, mínimo de 63 e máximo de 98 (dados não demonstrados). Deste total, houve predomínio do sexo feminino com 64,3\%. Na variável categórica estado civil do grupo em estudo, prevaleceram os solteiros com 57,1\%. O tempo médio

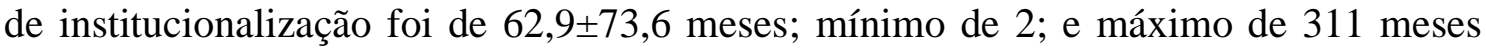
(dados não demonstrados), para ambos os sexos (Tabela 1).

Tabela 1. Caracterização de variáveis sociodemográficas de idosos institucionalizados da zona sul de São Paulo, 2015

\begin{tabular}{cccc}
\hline Variáveis categóricas & Classificação & $\mathbf{n}$ & \% \\
\hline \multirow{2}{*}{ Sexo } & Masculino & 15 & 35,7 \\
& Feminino & 27 & 64,3 \\
& & & \\
\multirow{2}{*}{ Estado civil } & Solteiro & 24 & 57,1 \\
& Casado & 2 & 4,8 \\
& Separado & 2 & 4,8 \\
& Viúvo & 14 & 33,3 \\
\hline
\end{tabular}

Os resultados da avaliação do estado nutricional dos idosos, segundo o IMC, mostrou que $35,7 \%$ dos participantes, estavam eutróficos. Em contrapartida, os resultados obtidos na Triagem mostraram que $62 \%$ apresentaram risco para desnutrição, e a média de escore foi de 10,64 $\pm 2,14$, (dados não demonstrados).

Quando avaliados pela MAN, verificou-se que 76,2\% do grupo apresentaram

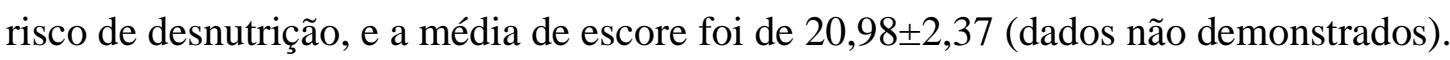

Dados da CB informaram que $42,9 \%$ dos participantes da pesquisa estavam eutróficos, e 4,7\% apresentaram desnutrição grave. 
$\mathrm{Na}$ avaliação da DCT, observou-se que $38 \%$ dos participantes foram classificados com desnutrição grave. Quando analisados os resultados de CP dos idosos, foi possível observar que $47,6 \%$ apresentaram valores adequados para a medida. Para os valores de $\mathrm{CC}$, a maioria deles, 55\%, apresentou risco aumentado para doenças metabólicas (Tabela 2).

Tabela 2. Caracterização do estado nutricional de acordo com avaliação antropométrica dos idosos institucionalizados da zona sul de São Paulo, 2015

\begin{tabular}{|c|c|c|c|}
\hline Variáveis categóricas & Classificação & $\mathbf{n}$ & $\%$ \\
\hline \multirow{3}{*}{$\operatorname{IMC}(n=42)$} & Magreza & 13 & 31 \\
\hline & Eutrofia & 15 & 35,7 \\
\hline & Excesso de peso & 14 & 33,3 \\
\hline \multirow[t]{2}{*}{ TRIAGEM (n= 42) } & Risco para desnutrição & 26 & 62 \\
\hline & Sem risco para desnutrição & 16 & 38 \\
\hline \multirow{3}{*}{ MAN $(n=42)$} & Desnutrição & 6 & 14,3 \\
\hline & Risco de desnutrição & 32 & 76,2 \\
\hline & Sem risco para desnutrição & 4 & 9,5 \\
\hline \multirow{6}{*}{$C B(n=42)$} & Desnutrição leve & 6 & 14,3 \\
\hline & Desnutrição moderada & 8 & 19 \\
\hline & Desnutrição grave & 2 & 4,7 \\
\hline & Eutrofia & 18 & 42,9 \\
\hline & Sobrepeso & 6 & 14,3 \\
\hline & Obesidade & 2 & 4,8 \\
\hline \multirow{6}{*}{ DCT $(n=42)$} & Desnutrição leve & 8 & 19 \\
\hline & Desnutrição moderada & 7 & 16,7 \\
\hline & Desnutrição grave & 16 & 38 \\
\hline & Eutrofia & 3 & 7,2 \\
\hline & Sobrepeso & 2 & 4,8 \\
\hline & Obesidade & 6 & 14,3 \\
\hline \multirow[t]{2}{*}{$\mathbf{C P}(\mathbf{n}=31)$} & Eutrófico & 20 & 47,6 \\
\hline & Desnutrição & 11 & 26,2 \\
\hline \multirow{3}{*}{$\mathrm{CC}(\mathrm{n}=36)$} & Risco aumentado & 23 & 55 \\
\hline & Risco & 3 & 7 \\
\hline & Sem risco & 10 & 23,8 \\
\hline
\end{tabular}




\section{Discussão}

Uma das limitações do presente estudo foi a dificuldade de acesso aos responsáveis e familiares para a assinatura do TCLE, o que influenciou na quantidade da amostra.

Nos resultados do trabalho foi possível observar que houve um predomínio de idosos do sexo feminino, solteiras, com idade avançada, com um longo tempo de institucionalização. Estudo semelhante realizado em instituição de longa permanência de São Paulo identificou que $82,4 \%$ dos idosos eram do sexo feminino (Volpini, \& Frangella, 2013). Outro estudo realizado em Uberlândia, MG, com idosos também institucionalizados obteve resultado semelhante do presente estudo para a média de idade, 79,5 anos, sendo a maioria do sexo feminino 62,7\% (Sousa, Mesquita, Pereira, \& Azeredo, 2014).

Segundo o Departamento de Informática do Sistema Único de Saúde (DATASUS), a expectativa de vida entre as mulheres é maior; portanto, este fato explica a maior proporção de mulheres idosas em relação aos homens (IBGE, 2006). Estudo semelhante realizado em duas ILPI existentes no município de Itaúna, MG, com 97 pessoas idosas observou um predomínio de $59 \%$ para o sexo feminino, com estado civil solteiro, com idade média de 77 anos. A maioria dos idosos era proveniente de domicílio próprio, onde moravam sozinhos e tinham dificuldades para realizar as atividades diárias. O tempo médio de institucionalização foi de 70 meses e variou entre 1 e 328 meses. Justamente pelo fato de não terem condições físicas e até mesmo psicossociais para viverem sozinhos, e por não terem suporte familiar de filhos ou cônjuges, se institucionalizaram (Lisboa, \& Chianca, 2012).

Analisando-se o IMC, nota-se que a maioria dos participantes foram classificados como eutróficos, corroborando os estudos de Galego, Sehnem, Novello, \& Santos (2013) que avaliaram o estado nutricional, valendo-se do IMC de idosos no Paraná, quando se constatou que a maioria dos avaliados apresentou eutrofia. Em relação à avaliação através da triagem e MAN, a maioria do grupo em estudo apresentou risco para desnutrição. Estes dados coincidem com os resultados de um estudo realizado na Espanha com 83 idosos institucionalizados, que verificou o estado nutricional, utilizando o IMC e a MAN e que revelou que, em ambos os métodos, a maioria dos residentes apresentaram risco para desnutrição (Martín, et al., 2012). 
Outro estudo realizado na República Checa em várias casas de repouso com 815 idosos mostrou que 10,2\% dos institucionalizados estavam desnutridos e 39,4\% estavam em risco de desnutrição (Rambousková, et al., 2013).

Estudo realizado em Roma, na Itália, com 100 idosos, que também utilizou a MAN e o IMC como parâmetro para avaliação nutricional, observou que houve uma prevalência de desnutrição e risco para desnutrição entre os participantes (Donini, Neri, Chiara, Poggiogalle, \& Muscaritoli, 2013). Utilizando o mesmo instrumento de avaliação, Pereira, et al. (2015) estudaram idosos residentes de 15 ILPI na cidade de Salvador e concluíram que, tanto pelo IMC quanto pela MAN, a maioria dos participantes estavam desnutridos e em risco de desnutrição.

Os resultados do presente estudo mostraram, pela classificação da $\mathrm{CB}$ e $\mathrm{CP}$, que a maior parte dos idosos estavam eutróficos. Dados da DCT relatou predomínio para desnutrição grave e na $\mathrm{CC}$ houve um predomínio de risco aumentado para doenças metabólicas. Idosos residentes na Turquia também estão em alto risco de adiposidade abdominal e doenças crônicas com base na $\mathrm{CC}$ e desnutridos, ou em risco de desnutrição, quando avaliados pela CB (Ongan, \& Rakicioglu, 2015). Estudo realizado com 344 idosos residentes em abrigos municipais do Rio de Janeiro apresentou resultado semelhante ao do presente estudo, mostrando que a maioria dos idosos estavam eutróficos pela classificação da CC e CP (Machado, \& Coelho, 2011).

Um estudo de intervenção realizado por Lee, et al. (2013), em Taiwan, com 92 idosos institucionalizados, obteve resultados diferentes do presente estudo para CB e $\mathrm{CP}$, cuja maioria apresentou risco para desnutrição. Os mesmos autores ressaltaram ainda que, após avaliação do estado nutricional, se houver risco, uma intervenção baseada na necessidade do idoso com uso de suplementação pode ser uma estratégia eficaz e útil para melhorar o estado nutricional e a qualidade de vida, devendo ser aplicada aos cuidados geriátricos institucionais, considerando-se que a proporção de idosos está aumentando nas populações do mundo.

Estudos indicam que os idosos institucionalizados apresentam maior prevalência de desnutrição do que os que vivem na comunidade (Freitas, Prado, Cação, Beretta, \& Albertini, 2015). Volpini e Frangella (2013) reforçaram a necessidade e a dificuldade da definição do diagnóstico nutricional em idosos institucionalizados, indicando o emprego de vários parâmetros antropométricos para se obter maior confiabilidade no diagnóstico nutricional da população idosa. 
Os mesmos autores ressaltaram ainda que os resultados obtidos de cada parâmetro devem ser avaliados com cautela e conjuntamente, principalmente com o profissional de nutrição para se delinear a condição nutricional do idoso. Dessa forma, o cuidado nutricional deve ser planejado de acordo com a avaliação nutricional, quando intervenções adaptadas e bem definidas podem ser desenvolvidas para esta população vulnerável (Ongan, \& Rakicioglu, 2015).

Os resultados do presente trabalho contribuíram para que a ILPI estudada pudesse identificar o estado nutricional dos idosos com diversos parâmetros para avaliação nutricional, permitindo o desenvolvimento de um plano terapêutico nutricional efetivo, apropriado e individualizado. Os dados coletados com a pesquisa contribuirão para a melhoria do atendimento nutricional na instituição, podendo servir de subsídio para uma intervenção diferenciada quando necessário. Para um acompanhamento detalhado e particularizado das necessidades nutricionais dos pacientes, sugere-se que os dados que compõem a avaliação sejam monitorados e reavaliados regularmente.

Por fim, é importante sugerir que novas pesquisas desta natureza sejam realizadas com o intuito de identificar e eventualmente alertar os profissionais de saúde e responsáveis sobre a importância de avaliar o estado nutricional de idosos institucionalizados.

\section{Conclusão}

Embora, alguns resultados estejam dentro dos limites nutricionais estabelecidos, é possível concluir que o risco de desnutrição e desnutrição foram condições de evidenciada importância, em vários idosos desta instituição. Esta pesquisa identificou a necessidade e, ao mesmo tempo, a dificuldade da definição do diagnóstico nutricional em idosos institucionalizados, sugerindo-se o emprego de vários parâmetros antropométricos, para se obter maior confiabilidade nos resultados encontrados nessa população.

Finalmente, de acordo com a associação entre estado nutricional e variáveis de diferentes dimensões, uma abordagem interdisciplinar no contexto da institucionalização é necessária, a fỉm de melhorar a saúde e qualidade de vida das pessoas idosas. 
Devido à complexidade de fatores envolvidos na avaliação e no diagnóstico nutricional de idosos, sugere-se que o trabalho em equipe com profissionais capacitados em diversas áreas disciplinares, permitindo uma visão abrangente, muito auxiliará na longevidade e na qualidade de vida desses indivíduos, especialmente quando institucionalizados.

\section{Referências}

Alves, L. C., Leimann, B. C. Q., Vasconcelos, M. E. L., Carvalho, M. S., Vasconcelos, A. G. G., Fonseca, T. C. O., Lebrão, M. L., \& Laurenti, R. (2005). A influência das doenças crônicas na capacidade funcional dos idosos do Município de São Paulo, Brasil. Rio de Janeiro, RJ: Caderno de Saúde Pública, 23(8), 1924-1930. Recuperado em 01 junho, 2015, de: http://dx.doi.org/10.1590/S0102-311X2007000800019.

Blackburn, G. L., \& Thornton, P. A. (1979). Nutritional assessment of the hospitalized patients. MedClin North Am, 63(5), 1103-1115. Recuperado em 01 junho, 2015, de: https://www.ncbi.nlm.nih.gov/pubmed/116095.

Brasil. (2003). Lei n. ${ }^{\circ} 10.741$, de 10 de outubro de 2003. Dispõe sobre o Estatuto do Idoso e dá outras providências. Diário Oficial da União, Brasília, DF, 3 out. 2003. Seção1, 1. Recuperado em 01 junho, 2015, de: http://www.planalto.gov.br/ccivil_03/leis/2003/L10.741.htm.

Brasil. (1994). Lei n. 8.842, de 4 de janeiro de 1994. Dispõe sobre a Política Nacional do Idoso, cria o Conselho Nacional do Idoso e dá outras providências. Diário Oficial da União, Brasília, DF, 5 jan. 1994. Recuperado em 01 junho, 2015, de: http://www.planalto.gov.br/ccivil_03/leis/L8842.htm.

Brasil. (2012). Ministério do Planejamento, Orçamento e Gestão. Relatório de Gestão 2011. Instituto Brasileiro de Geografia e Estatística, IBGE. Brasília, DF. Recuperado em 01 junho, 2015, de:

https://ww2.ibge.gov.br/home/disseminacao/prestacaodecontas/relatgestao2011.pdf.

Chumlea, W. C., Roche, A. F., \& Steinbaugh, M. L. (1985). Estimating stature from knee height for person 60 to 90 year of age. Jama, 33(2), 116-120. Recuperado em 01 junho, 2015, de: https://www.ncbi.nlm.nih.gov/pubmed/3968366.

Chumlea, W. C., Guo, S. S., Vellas, B., \& Guigoz, Y. (1995). Techniques of assessing muscle mass and function (sarcopenia) for epidemiological studies of the elderly. Journals of Gerontology Series. Germany, 50(Spec No), 45-51. Recuperado em 01 junho, 2015, de: https://www.ncbi.nlm.nih.gov/pubmed/7493217.

Donini, L. M., Neri, B., Chiara, S., Poggiogalle, E., \& Muscaritoli, M. (2008). Nutritional Care in a Nursing Home in Italy, 8(2), e55804. Recuperado em 01 junho, 2015, de: https://doi.org/10.1371/journal.pone.0055804. 
Freitas, A. F., Prado, M. A., Cação, J. C., Beretta, D., \& Albertini, S. (2015). Sarcopenia e estado nutricional de idosos: uma revisão da literatura. Arq. Ciênc. Saúde, 22(1), 0913. Recuperado em 01 junho, 2015, de: doi: https://doi.org/10.17696/23183691.22.1.2015.19.

Frisancho, A. R. (1984). New standards of weight and body composition by frame size and height for assessment of nutritional status of adults and the elderly. Am. J. Clin. Nutr., 40(4), 808-819. Recuperado em 01 junho, 2015, de: https://www.ncbi.nlm.nih.gov/pubmed/6486088.

Galego, B. V., Sehnem, R. C., Novello, D., \& Santos, E. F. dos. (2013). Mini-Avaliação Nutricional (MAN) e Índice de Massa Corporal (IMC) e sua associação com hipertensão arterial em idosos fisicamente ativos. Uniciências, 17(1), 11-15. Recuperado em 01 junho, 2015, de: doi: http://dx.doi.org/10.17921/1415$5141.2013 \mathrm{v} 17 \mathrm{n} 1 \mathrm{p} \% 25 \mathrm{p}$.

Guigoz, Y., Vellas, B., \& Garry, P. J. (1994). Mini nutritional assessment: A practical assessment tool for grading the nutritional state of elderly patients. Facts and research in gerontology,4(Suppl.2), 15-59. Recuperado em 01 junho, 2015, de: https://ci.nii.ac.jp/naid/10030728344/.

IBGE. (2006). Instituto Brasileiro de Geografia e Estatística. Recuperado em 19 outubro, 2014, de: http://www.ibge.gov.br/ibgeteen/datas/idoso/politica_do_idoso_no_brasil.htm.

Lee, L-C., Alan C. T., Wang, J-Y., Hurng, B-S., Hsu, H-C., \& Tsai, H-J. (2013). Needbased intervention is an effective strategy for improving the nutritional status of older people living in a nursing home: A randomized controlled trial. International Journal of Nursing Studies, 50(12), 1580-1588. Recuperado em 01 junho, 2015, de: doi: 10.1016/j.ijnurstu.2013.04.004.

Lisboa, C. R., \& Chianca, T. C. M. (2012). Perfil epidemiológico, clínico e de independência funcional de uma população idosa institucionalizada. Rev. Bras. Enferm. Brasília, 65(3), 482-487. Recuperado em 01 junho, 2015, de: http://dx.doi.org/10.1590/S0034-71672012000300013.

Machado, R. S. P., \& Coelho, M. A. S. C. (2011). Risk of malnutrition among brazilian institutionalized elderly: a study with the mini nutritional assessment (MNA) questionnaire. The Journal of Nutrition, Health \& Aging, 15(7), 532-535. Recuperado em 01 junho, 2015, de: https://www.ncbi.nlm.nih.gov/pubmed/21808930.

Martín, M. ${ }^{a}$ A. C., Ortega, S. B., Rodríguez, L. D., Muiño, C., Silleras, B. M., \& Río, M. ${ }^{a}$ P. R. D. (2012). Presencia de malnutrición y riesgo de malnutrición en ancianos institucionalizados con demencia en función del tipo y estadío evolutivo. Nutr. Hosp, 27(2), 434-440. Recuperado em 01 junho, 2015, de: http://scielo.isciii.es/scielo.php?script=sci_arttext\&pid=S0212-16112012000200013.

Menezes, R. L., \& Bachion, M. M. (2012). Condições visuais autorrelatadas e quedas em idosos institucionalizados. Rev. Bras. Oftalmol., 71(1), 23-27. Recuperado em 01 junho, 2015, de: http://dx.doi.org/10.1590/S0034-72802012000100005.

NSI. (1994). Nutrition Screening Initiative. Incorporation nutrition screening and interventions into medical practice: A monograph for physicians. Washington, DC. 
Ongan, D., \& Rakicioglu, N. (2015). Nutritional status and dietary intake of institutionalized elderly in Turkey: A cross-sectional, multi-center, country representative study. Archives of Gerontology and Geriatrics, 61(2), 271-276. Recuperado em 01 junho, 2015, de: doi: 10.1016/j.archger.2015.05.004.

Papparotto, C., Bidoli, E., \& Palese, A. (2013). Risk factors associated with alnutrition in older adults living in Italian nursing homes: A cross-sectional study. Research in Gerontological Nursing, 6(3), 187-197. Recuperado em 01 junho, 2015, de: doi: 10.3928/19404921-20130528-01.

Paz, R. C., Fazzio, D. M. G., \& Santos, A. L. B. (2012). Avaliação nutricional em idosos institucionalizados. Revisa, 1(1), 9-18. Recuperado em 01 junho, 2015, de:

Pereira, M. L. A. S., Moreira, P. A., Oliveira, C. C., Roriz, A. K. C., Amaral, M. T. R., Mello, A. L., \& Ramos, L. B. (2015). Nutritional status of institutionalized elderly Brazilians: a study with the Mini Nutritional Assessment. Nutr. Hosp., 31(3), 11981204. Recuperado em 01 junho, 2015, de: file://C:/Users/Dados/Downloads/6-11-1PB.pdf.

Rambousková, J., Slavíková, M., Krsková, A., Bohumír, P., Anděl M., \& Dlouhý, P. (2013). Nutritional Status Assessment of Institutionalized Elderly in Prague, Czech Republic. Ann. Nutr. Metab., 62(3), 201-206. Recuperado em 01 junho, 2015, de: doi: $10.1159 / 000346038$.

Ramos, L. J., Pizzato, A. C., Ettrich, B., Melnik, C. S., \& Goldim, J. R. (2012). Aspectos éticos e nutricionais em uma amostra de idosos institucionalizados e não institucionalizados. Rev. HCPA, 32(2), 223-226. Recuperado em 01 junho, 2015, de: http://hdl.handle.net/10183/158765.

Sousa, K. T., Mesquita, L. A. S., Pereira, L. A., \& Azeredo, C. M. (2014). Baixo peso e dependência funcional em idosos institucionalizados de Uberlândia (MG), Brasil. Ciência \& Saúde Coletiva, 19(8), 3513-3520. Recuperado em 01 junho, 2015, de: https://doi.org/10.1590/1413-81232014198.21472013.

Souza, C. C., Valmorbida, L. A., Oliveira, J. P., Borsatto, A. C., Lorenzini, M., Knorst, M. R., MELO, D., Creutzberg, M., \& Resende, T. de L. (2013). Mobilidade funcional em idosos institucionalizados e não institucionalizados. Rio de Janeiro, RJ: Rev. Bras. Geriatr. Gerontol., 16(2), 285-293. Recuperado em 01 junho, 2015, de: http://dx.doi.org/10.1590/S1809-98232013000200008.

Volpini, M. M., \& Frangella, V. S. (2013). Avaliação nutricional de idosos institucionalizados. Einstein, 11(1), 32-40. Recuperado em 01 junho, 2015, de: http://apps.einstein.br/revista/arquivos/pdf/2495-32-40_port.pdf.

WHO. (2000). World Health Organization. Obesity: preventing and managing the global epidemic: Report of a WHO consultation on obesity. Geneva, Suisse: Technical Report Series, 894. Recuperado em 01 junho, 2015, de: http://www.who.int/nutrition/publications/obesity/WHO_TRS_894/en/.

WHO. (1995). World Health Organization. World Health Organization. Physical Status: The use and interpretation of anthropometry. Genebra, Suisse: World Health Organization. Recuperado em 01 junho, 2015, de: http://apps.who.int/iris/handle/10665/37003.

WHO. (2011). World Health Organization Global Health and Aging. Recuperado em 12 julho, 2015, de: http://www.who.int/ageing/publications/global_health.pdf. 
Recebido em 12/11/2015

Aceito em 30/01/2016

Dalvineia Carvalho Santana - Graduação em Nutrição, Centro Universitário Adventista de São Paulo.

E-mail: neia.juara@hotmail.com

Daiane Soares Macedo - Graduação em Nutrição, Centro Universitário Adventista de São Paulo.

E-mail: dsmacedo@hotmail.com

Nyvian Alexandre Kutz - Graduação em Nutrição, Centro Universitário Adventista de São Paulo.

E-mail: nyviankutz@hotmail.com

Marcia Maria Hernandes de Abreu de Oliveira Salgueiro - Graduação em Nutrição. Mestrado e Doutorado em Saúde Pública, Faculdade de Saúde Pública, Universidade de São Paulo, USP. Professor Doutor, Curso de Graduação em Nutrição, Centro Universitário Adventista de São Paulo, do Mestrado em Promoção da Saúde e Coordenadora do Curso de Pós-Graduação em Nutrição Clínica Ambulatorial do Centro Universitário Adventista de São Paulo.

E-mail: marciasalgueironutricionista@yahoo.com.br 